Boise State University

ScholarWorks

$1-1-2018$

\title{
A Framework for Relating the Structures and Recovery Statistics in Pressure Time-Series Surveys for Dust Devils
}

Brian Jackson

Boise State University

Ralph Lorenz

Johns Hopkins University Applied Physics Lab

Karan Davis

Boise State University

\section{Publication Information}

Jackson, Brian; Lorenz, Ralph; and Davis, Karan. (2018). "A Framework for Relating the Structures and Recovery Statistics in Pressure

Time-Series Surveys for Dust Devils". Icarus, 299, 166-174. http://dx.doi.org/10.1016/j.icarus.2017.07.027

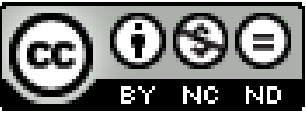

This is an author-produced, peer-reviewed version of this article. (C) 2018, Elsevier. Licensed under the Creative Commons Attribution NonCommercial No Derivatives 4.0. http://creativecommons.org/licenses/by-nc-nd/4.0/. The final, definitive version of this document can be found online at Icarus, doi: 10.1016/j.icarus.2017.07.027 


\section{A Framework for Relating the Structures and Recovery Statistics in Pressure Time-Series Surveys for Dust Devils}

Brian Jackson $^{\text {a }}$, Ralph Lorenz ${ }^{\mathrm{b}}$, Karan Davis ${ }^{\mathrm{a}}$

${ }^{a}$ Department of Physics, Boise State University, 1910 University Drive, Boise, ID 83725-1570, USA

${ }^{\mathrm{b}}$ Johns Hopkins University Applied Physics Lab, 11100 Johns Hopkins Road, Laurel, Maryland 20723-6099, USA

Number of pages: 39

Number of figures: 10

Preprint submitted to Icarus 
Proposed Running Head:

De-biasing Dust Devil Surveys

Please send Editorial Correspondence to:

Brian Jackson

Department of Physics,

Boise State University

1910 University Drive

Boise, ID 83725-1570, USA

Email: bjackson@boisestate.edu

Phone: (208) 426-3723 


\section{ABSTRACT}

Dust devils are likely the dominant source of dust for the martian atmosphere, but the amount and frequency of dust-lifting depend on the statistical distribution of dust devil parameters. Dust devils exhibit pressure perturbations and, if they pass near a barometric sensor, they may register as a discernible dip in a pressure time-series. Leveraging this fact, several surveys using barometric sensors on landed spacecraft have revealed dust devil structures and occurrence rates. However powerful they are, though, such surveys suffer from non-trivial biases that skew the inferred dust devil properties. For example, such surveys are most sensitive to dust devils with the widest and deepest pressure profiles, but the recovered profiles will be distorted, broader and shallow than the actual profiles. In addition, such surveys often do not provide wind speed measurements alongside the pressure time series, and so the durations of the dust devil signals in the time series cannot be directly converted to profile widths. Fortunately, simple statistical and geometric considerations can de-bias these surveys, allowing conversion of the duration of dust devil signals into physical widths, given only a distribution of likely translation velocities, and the recovery of the underlying distributions of physical parameters. In this study, we develop a scheme for de-biasing such surveys. Applying our model to an in-situ survey using data from the Phoenix lander suggests a larger dust flux and a dust devil occurrence rate about ten times larger than previously inferred. Comparing our results to dust devil track surveys suggests only about one in five low-pressure cells lifts sufficient dust to leave a visible track.

Keywords: Mars, atmosphere ; Mars, surface ; Earth 


\section{Introduction}

Dust devils are small-scale (a few to tens of meters) low-pressure vortices rendered visible by lofted dust. They usually occur in arid climates on the Earth and ubiquitously on Mars. Martian dust devils have been studied with orbiting and landed spacecraft and were first identified on Mars using images from the Viking Orbiter (Thomas and Gierasch, 1985). On Mars, dust devils may dominate the supply of atmospheric dust and influence climate (Basu, 2004), pose a hazard for human exploration (Balme and Greeley, 2006), and have lengthened the operational lifetime of Martian rovers (Lorenz and Reiss, 2014). On the Earth, dust devils significantly degrade air quality in arid climates (Gillette and Sinclair, 1990) and may pose an aviation hazard (Lorenz and Myers, 2005).

The dust-lifting capacity of dust devils seems to depend sensitively on their structures, in particular on the pressure wells at their centers (Neakrase et al., 2006), so the dust supply from dust devils on both planets may be dominated by the seldom observed, most vigorous devils. Thus, elucidating the origin, evolution, and population statistics of dust devils is critical for understanding important terrestrial and Martian atmospheric properties.

Studies of Martian dust devils have been conducted through direct imaging of the devils and identification of their tracks on Mars' dusty surface (Balme and Greeley, 2006). Studies with in-situ meteorological instrumentation have also identified dust devils, either via obscuration of the Sun by the dust column (Zorzano et al., 2013) or their pressure signals (Ellehoj et al., 2010). Studies have also been conducted of terrestrial dust devils and frequently involve in- 
person monitoring of field sites. Terrestrial dust devils are visually surveyed (Pathare et al. 2010), directly sampled (Balme et al., 2003a), or recorded using in-situ meteorological equipment (Sinclair, 1973; Lorenz, 2012).

As noted in Lorenz (2009), in-person visual surveys are likely to be biased toward detection of larger, more easily seen devils. Such surveys would also fail to recover dustless vortices (Lorenz and Jackson, 2015). Recently, terrestrial surveys similar to Martian dust devil surveys have been conducted using in-situ single barometers (Lorenz, 2012, 2014; Jackson and Lorenz, 2015) and photovoltaic sensors (Lorenz and Jackson, 2015). These sensor-based terrestrial surveys have the advantage of being directly analogous to Martian surveys and are cost-effective compared to the in-person surveys.

Single-barometer surveys have been successful on both planets in identifying and elucidating the properties of dust devils (Murphy et al., 2016). In this kind of survey, a sensor is deployed in-situ and records a pressure time series at a sampling rate $\geq 1 \mathrm{~Hz}$. Since it is a low-pressure convective vortex, the nearby passage of a dust devil will register as pressure dip discernible against a background ambient (but not necessarily constant) pressure. Figure 1 adapted from (Jackson and Lorenz, 2015) shows a time-series with a typical dust devil signal.

However, single-sensor barometer surveys also suffer from important biases. Foremost among these biases is the fact that a pressure dip does not necessarily correspond to a dust-lofting vortex. Indeed, recent studies Steakley and Murphy, 2014) suggest pressure dips are often unaccompanied by lofted dust, likely because the attendant wind velocities are not sufficient to lift dust. The problem of identifying dustless vortices can be mitigated if barometers are 


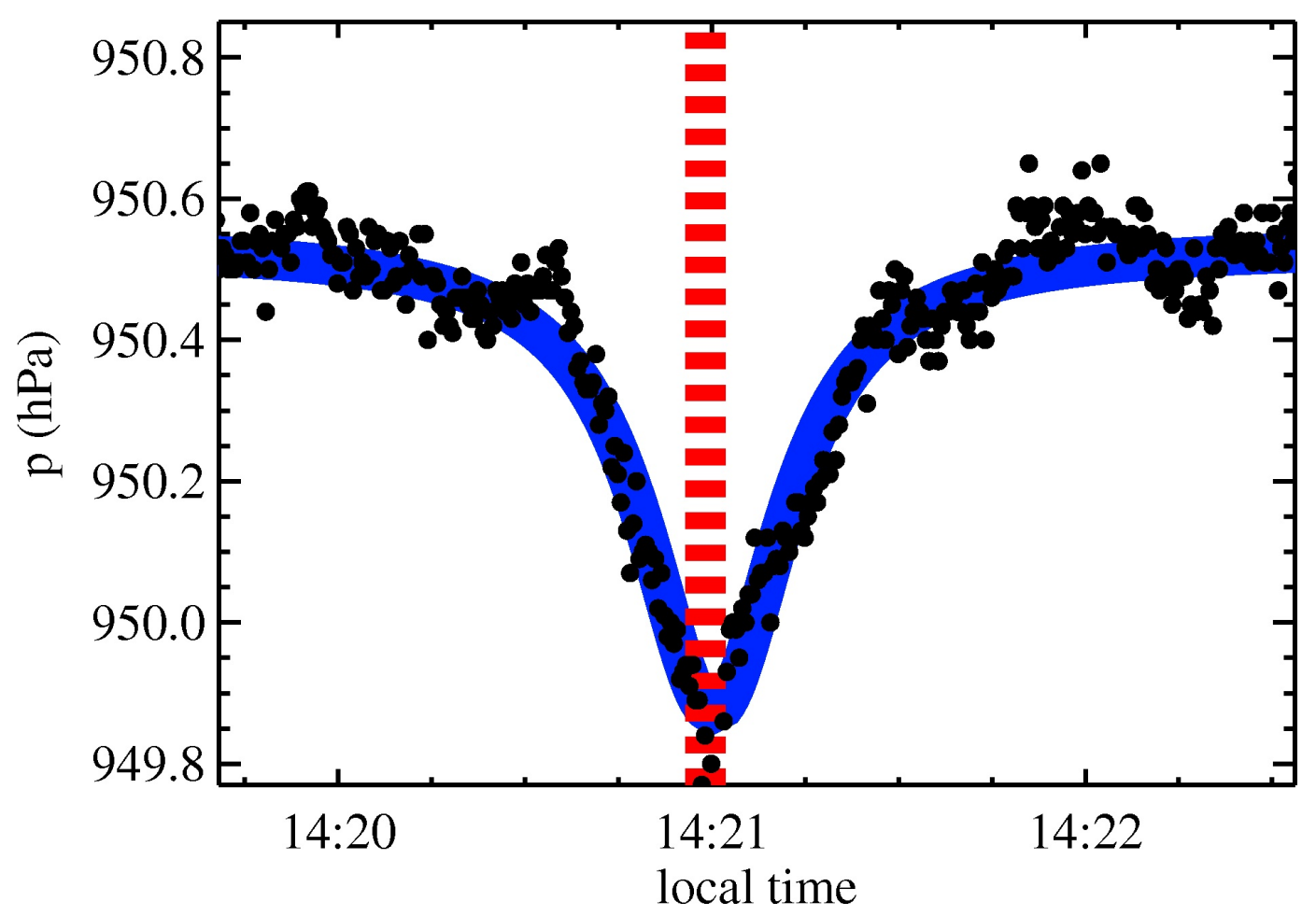

Fig. 1. Example dust devil profile from Jackson and Lorenz (2015). The black dots show the pressure $p$ measurements (in hectoPascal hPa) as a function of local time in hours. The blue line shows the best-fit profile model, and the red, dashed line the profile center. Both lines are made extra thick only to enhance visibility.

deployed alongside solar cells, which can register obscuration by dust as long as the dust-lifting devil passes between the sensor and the Sun Lorenz and Jackson, 2015).

Another key bias is the "miss distance" effect: a fixed barometric sensor is very likely to pass through a dust devil some distance from its center. Since the pressure perturbation falls off with distance, the deepest point in the observed pressure profile will almost always be less than the pressure dip at the devil's center. The observed shape of the profile will be distorted as well. Additional biases can also influence the inferred statistical properties: noise in the pressure time series from a barometer may make more difficult detection 
of a dust devils with smaller pressure perturbations, depending on the exact detection scheme.

However, simple geometric considerations can mitigate the influence of the miss distance effect, allowing single-barometer surveys to be statistically corrected. Such statistical considerations are key for understanding the populationaveraged influence of dust devils (Lorenz and Jackson, 2016). In this study, we present a model for correcting the miss distance effect. This study is motivated by Lorenz (2014), but, where that study used a numerical simulation to investigate biases in the recovered population of dust devil properties, we employ an analytic framework that provides more direct insight into the problem.

In Section 2, we develop our statistical model and describe how the geometry of encounter both biases and distorts the recovered parameters. In Section 3, we conduct a preliminary application of our model to data from a dust devil survey, and in Section 4, we discuss some of the limitations of our model, ways to improve it, and future work.

\section{Formulating the Statistical Model}

To develop a model for the recovery biases and signal distortions induced by the miss distance effect, we will make the following assumptions:

(1) Each dust devil pressure profile has a well-defined, static radial profile, which follows a Lorentzian:

$$
P(r)=\frac{P_{\mathrm{act}}}{1+\left(2 r / \Gamma_{\mathrm{act}}\right)^{2}},
$$

where $r$ is the distance from the devil center, $P_{\text {act }}$ the actual pressure 
depth at the devil's center, and $\Gamma_{\text {act }}$ the profile full-width at half-max. Alternative profiles have been suggested, (e.g., Burgers-Rott and Vatistas profiles - Lorenz, 2014; Kurgansky et al., 2016), but using a different profile in our analysis would not qualitatively change our results. For instance, a profile that fell off more slowly with radial distance would less significantly bias the recovered devil population toward larger devils (Section 2.3), but the bias would persist.

(2) The dust devil center travels at a translation velocity $v$, which is constant in magnitude and direction. In reality, a devil's trajectory can be complex, even encountering a sensor multiple times and/or a devil may consist of multiple convective cores, consequently producing complex pressure signals (Lorenz, 2013). The time it takes for a devil to travel a distance equal to its own diameter (i.e., full-width/half-max) $\Gamma_{\text {act }}$ is $\tau_{\text {act }}=\Gamma_{\text {act }} / v$. By contrast, the pressure signal registered by a devil is observed to have a width in time $\tau_{\text {obs }}=\Gamma_{\text {obs }} / v$, where $\Gamma_{\text {obs }}$ is the inferred width of the devil's profile.

(3) Dust devil velocities follow a smooth distribution $\rho(v)$ such that the small fraction of dust devils that have velocities between $v$ and $v+d v$ is $\rho(v) d v$. Since pressure time-series register devils as signals in time, converting from the devil profile duration to its width requires accounting for the travel velocity.

(4) A dust devil appears and disappears instantaneously, traveling a distance $v L$ over its lifetime $L$. As pointed out by Lorenz (2013), $L$ seems to depend on dust devil diameter $D$ as $L=40 \mathrm{~s}(D / \mathrm{m})^{2 / 3}$, with diameter in meters. We assume $D=\Gamma_{\text {act }}$ (Vatistas et al., 1991).

(5) There are minimum and maximum pressure profile depths that can be recovered by a survey, $P_{\text {th }}$ and $P_{\max }$, respectively. $P_{\text {th }}$ may be set by the 
requirement that a pressure signal exceeds some minimum threshold set by the noise in the datastream, while basic thermodynamic limitations likely restrict the maximum pressure depth for a devil (Rennó et al. 1998). Likewise, the profile widths must fall between $\Gamma_{\text {th }}$ and $\Gamma_{\max }$, possibly set by the ambient vorticity field in which a devil is embedded (Rennó and Bluestein, 2001) and/or the depth of the planetary boundary layer (Fenton and Lorenz, 2015). The two sets of limits may not be related, i.e. devils with $P_{\max }$ do not necessarily have widths $\Gamma_{\max }$. As it turns out, our results are not sensitive to the precise values for each of these limits.

(6) The two-dimensional distribution of $P_{\text {act }}$ and $\Gamma_{\text {act }}, \rho\left(P_{\text {act }}, \Gamma_{\text {act }}\right)$, is integrable and differentiable. The same is true for the distributions of observed dust devil parameters, $\rho\left(P_{\text {obs }}, \Gamma_{\text {obs }}\right)$. It is also important to note that all the distributions shown here are differential distributions and not cumulative (Lorenz, 2011).

(7) The uncertainties on the profile depth and width estimated for a dust devil are negligible. In Jackson and Lorenz (2015), for example, uncertainties on $P_{\text {obs }}$ were about an order of magnitude less than the inferred $P_{\text {obs }}$ value for a detected devil, with uncertainties on $\Gamma_{\text {obs }}$ at least a factor of three smaller. Robust recovery of a devil against noise requires relatively small uncertainties.

We can relate the geometry of an encounter directly to the observed profile parameters, and Figure 2 illustrates a typical encounter. As a devil passes the barometer, it will have a closest approach distance $b$, and the radial distance between devil center and sensor $r(t)=\sqrt{b^{2}+(v t)^{2}}$, where time $t$ runs from negative to positive values. The fact that $b$ is usually greater than zero biases the devils that are detected and the way in which their pressure signals reg- 


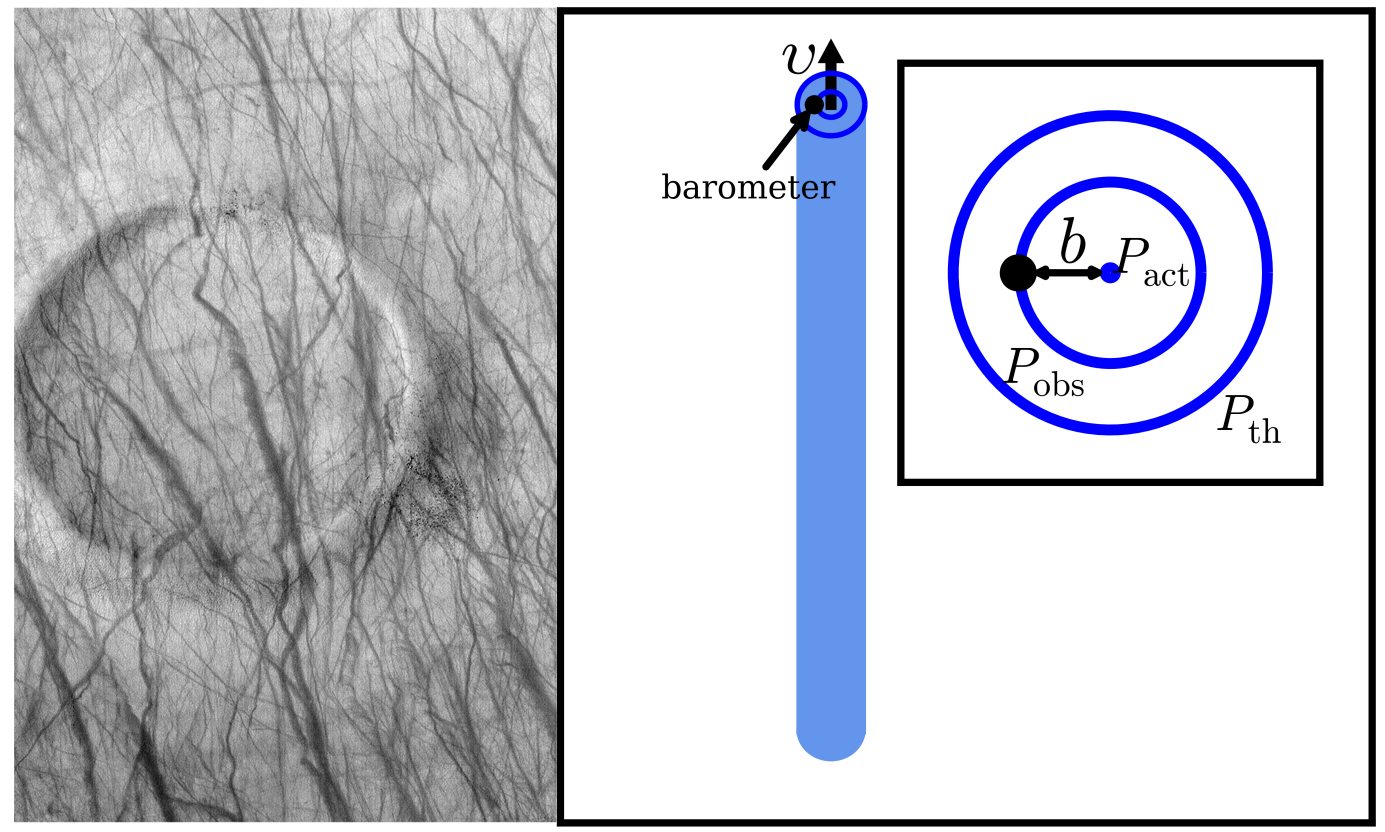

Fig. 2. (Left) Mars Global Surveyor Mars Orbiter Camera image of crater showing dust devil tracks. The image covers an area $3 \mathrm{~km}$ $(1.9 \mathrm{mi})$ wide, is illuminated from the upper left, and is available at http://www.msss.com/mars_images/moc/2003/12/02/2003.12.02.R0901707.gif.

(Right) Geometry of dust devil encounter. The blue circles show pressure contours, the y-axis runs along the devil's translational velocity vector measured relative to the sensor $v$, and $b$ is the closest approach distance between the barometer and the devil center. The shaded blue region shows the area of the surface carved out by the traveling $P_{\text {th }}$ contour. The inset shows a close-up of the encounter.

ister. First, we discuss how to convert the distribution of observed dust devil durations to inferred diameters. Next, we formulate the recovery biases and signal distortions resulting from the miss distance effect. 


\subsection{Converting Durations to a Distribution of Diameters}

Very often, the velocity with which a dust devil is traveling as it registers a pressure signal is unknown, which, unfortunately, means the duration of a devil in a time-series $\tau$ cannot be directly converted to a profile width $\Gamma$ (both the observed and actual values are afflicted in the same way with this uncertainty). Via field experiment, Balme et al. (2012) found that dust devils tend to follow the ambient wind field, and Lorenz (2016) argued that the departure in migration direction from the ambient wind corresponds to an additional translational velocity vector about $2 \mathrm{~m} / \mathrm{s}$ in magnitude with a random azimuth. Even without a directly measured translation velocity, we can convert statistically from $v$ and $\tau$ values to $\Gamma$ values.

If we have a collection of discretely measured dust devil durations $\tau_{\mathrm{i}}$ from a time-series analysis and an expected distribution of wind speeds, we can ask what is the probability that the ith dust devil had a $\Gamma$ value between $\Gamma$ and $\Gamma+d \Gamma$. Elementary statistics indicates that this distribution $\rho(\Gamma)$ is

$$
\rho(\Gamma)=\left(\frac{d v}{d \Gamma}\right)_{\mathrm{i}}\left(\frac{d N}{d v}\right)=\tau_{\mathrm{i}}^{-1} \rho\left(v=\Gamma / \tau_{\mathrm{i}}\right),
$$

where $\rho(v)$ is the probability density for the wind speed.

We can also convert the joint distribution for $v$ and $\tau$ to a distribution of $\Gamma$ values:

$$
\rho(\Gamma)=\frac{d}{d \Gamma} \int_{D_{\Gamma}} \rho(v, \tau) d v d \tau
$$

where $D_{\Gamma}$ is the region in $(v, \tau)$-space illustrated in Figure 3 (a).

From the figure, we can see that when $\Gamma \leq v_{\max } \tau_{\min }$, the integration limits are $\tau \in\left[\tau_{\min }, \max \left(\tau_{\max }, \Gamma / v_{\min }\right)\right]$ and $v \in\left[v_{\min }, \Gamma / \tau\right]$. When $\Gamma>v_{\max } \tau_{\min }$, 

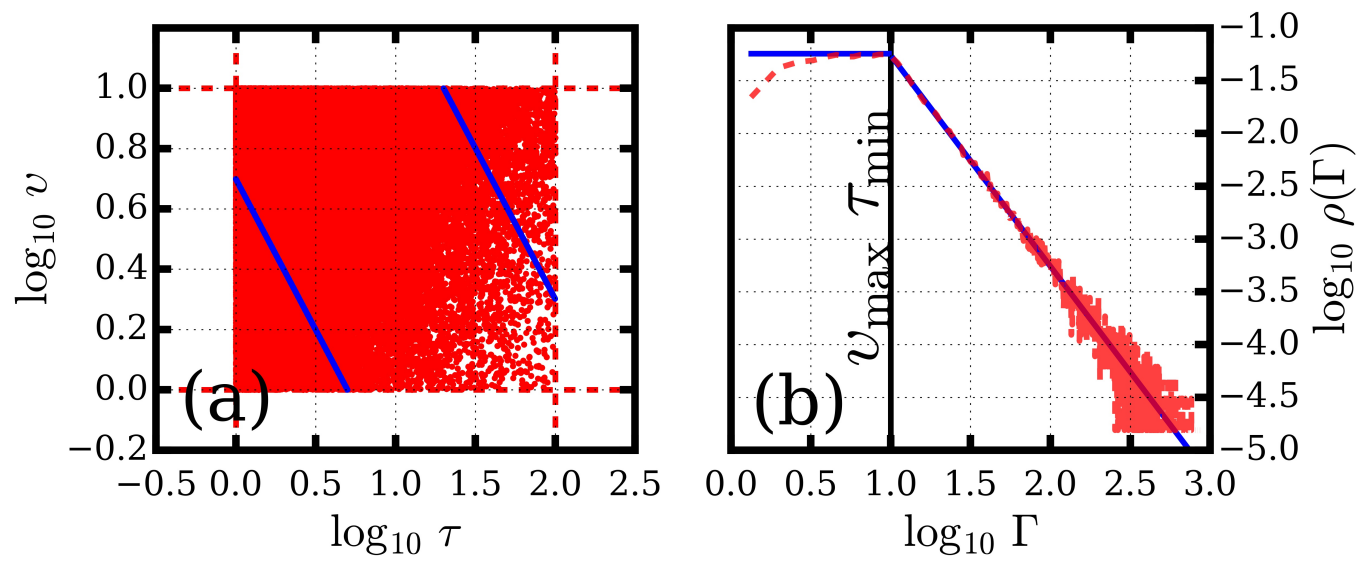

Fig. 3. (a) A distribution of $v$ - (uniform) and $\tau$ values $\left(\sim \tau^{-2}\right)$, shown as red dots, along with the integration tracks for calculating $\rho(\Gamma)$, shown as blue curves. (b) The resulting $\rho(\Gamma)$. The dashed, red line shows the direct numerical conversion of the $v-\tau$ distributions from (a), while the solid, blue lines show the analytic prediction. The discrepancies between the two curves at the left and right ends result from binning and sampling distortions.

$\tau \in\left[\Gamma / v_{\max }, \max \left(\tau_{\max }, \Gamma / v_{\min }\right)\right]$ and $v \in\left[v_{\min }, \Gamma / \tau\right]$

In Section 3, we calculate this integral numerically using real data, but as an example, consider a simple differential distribution:

$$
\rho(v, \tau)=k \tau^{-\alpha}
$$

for which velocities are uniformly distributed. The resulting distribution of $\Gamma$ values has a complicated, piece-wise form described in the Appendix and is shown in Figure 3 (b) for $\alpha=2$. 


\subsection{The Signal Distortion}

The deepest point observed in the pressure profile $P_{\text {obs }}$ is given by

$$
P_{\mathrm{obs}}=\frac{P_{\mathrm{act}}}{1+\left(2 b / \Gamma_{\mathrm{act}}\right)^{2}}
$$

Clearly, unless $b=0, P_{\text {obs }}<P_{\text {act }}$. Likewise, non-central encounters will distort the profile full-width/half-max, giving a full-width/half-max $\Gamma_{\text {obs }}$.

The observed pressure signal drops to half its value at a time $t= \pm \frac{1}{2} \tau_{\text {obs }}=$ $\pm \frac{1}{2} \Gamma_{\text {obs }} / v$ by definition. At these times, the center of the devil is a radial distance from the barometer $r\left(t= \pm \tau_{\text {obs }} / 2\right)=\sqrt{b^{2}+\left(\frac{1}{2} \Gamma_{\text {obs }}\right)^{2}}$ and

$$
P(r)=\frac{1}{2} P_{\mathrm{obs}}=\frac{1}{2} \frac{P_{\mathrm{act}}}{1+\left(2 b / \Gamma_{\mathrm{act}}\right)^{2}}=\frac{P_{\mathrm{act}}}{1+\left(2 r\left( \pm \tau_{\mathrm{obs}} / 2\right) / \Gamma_{\mathrm{act}}\right)^{2}} .
$$

Solving for $\Gamma_{\text {obs }}$ gives

$$
\Gamma_{\mathrm{obs}}^{2}=\Gamma_{\mathrm{act}}^{2}+(2 b)^{2} .
$$

Figure 4 shows how a non-central encounter modifies the observed pressure profile. $\Gamma_{\text {obs }}$ for the green curve with $b=\Gamma_{\text {act }}$ is $\sqrt{1+2^{2}} \approx 2.2$ times larger than that for the blue curve with $b=0$. We call this modification the signal distortion. Combining the Equations 5 and 7 gives the following useful expression:

$$
P_{\mathrm{obs}} \Gamma_{\mathrm{obs}}^{2}=P_{\mathrm{act}} \Gamma_{\mathrm{act}}^{2} \text {. }
$$

We can solve Equation 5 for $b$ :

$$
b=\left(\frac{\Gamma_{\mathrm{act}}}{2}\right) \sqrt{\frac{P_{\mathrm{act}}-P_{\mathrm{obs}}}{P_{\mathrm{obs}}}} .
$$

A single barometer at a fixed location can sense a dust devil only over a certain area, spanning a maximum radial distance $b_{\max }$, beyond which devils 


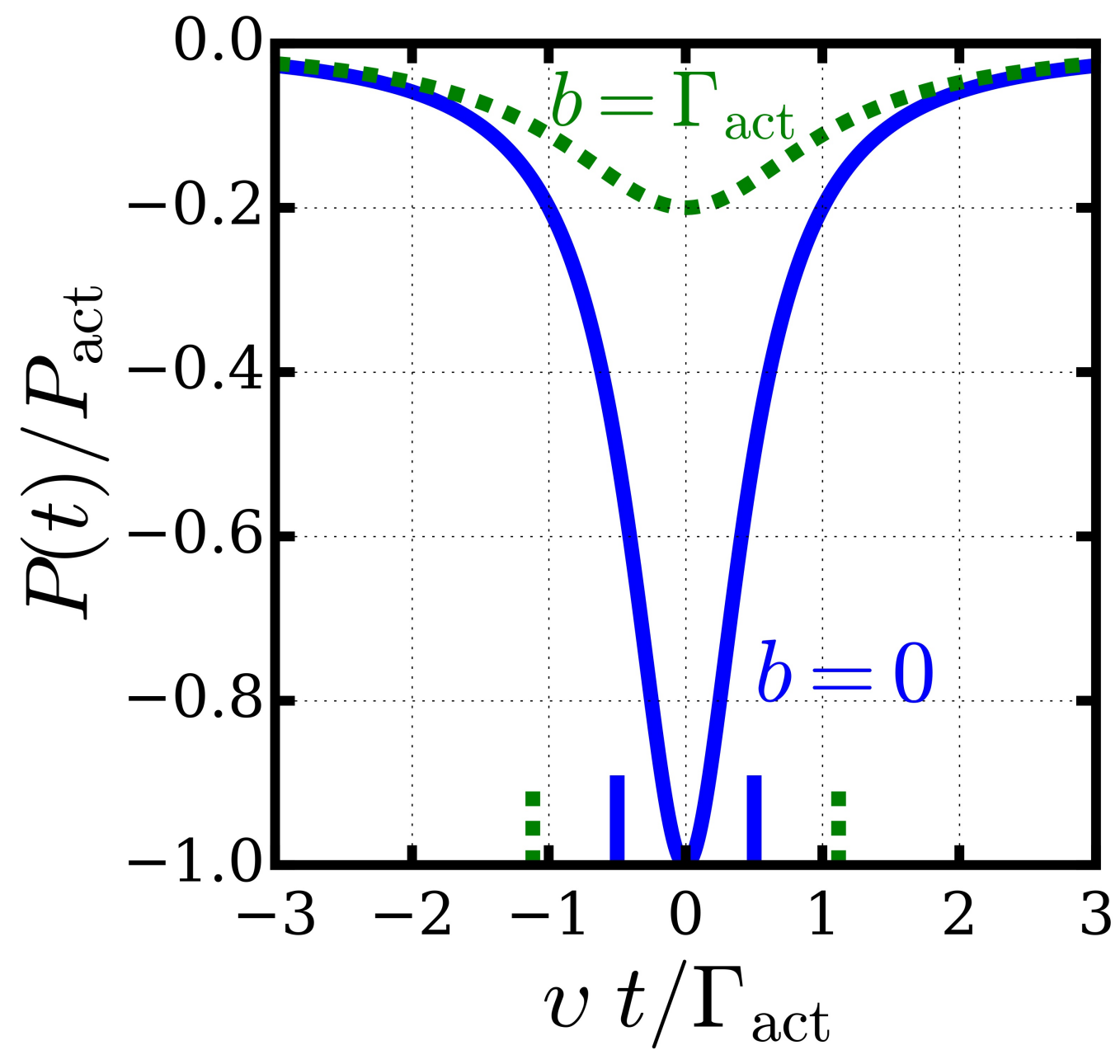

Fig. 4. Dust devil profiles for $b=0$ and $b=\Gamma_{\text {act }}$, i.e. for a closest approach equal to the profile's diameter. The vertical solid, blue and dashed, green lines at the bottom of the plot show the respective $\Gamma_{\text {obs }}$ values.

will produce pressure signals smaller than the detection threshold, $P_{\text {obs }}<P_{\text {th }}$ :

$$
b_{\max }=\left(\Gamma_{\mathrm{act}} / 2\right) \sqrt{\frac{P_{\mathrm{act}}-P_{\mathrm{th}}}{P_{\mathrm{th}}}} .
$$

We can use the encounter geometry to model the statistical probability for $P_{\text {obs }}$ and $\Gamma_{\text {obs }}$ to fall within a certain range of values, given a distribution of $P_{\text {act }}$ and $\Gamma_{\text {act }}$ values. The probability density for passing between $b$ and $b+d b$ of a devil is $d p(b)=2 b d b / b_{\max }^{2}$ for $b \leq b_{\max }$. This expression allows us to 
calculate the average miss distance for a given dust devil:

$$
\left\langle b / \Gamma_{\text {act }}\right\rangle=\int b / \Gamma_{\text {act }} d p=\frac{2}{3} b_{\max } / \Gamma_{\text {act }}
$$

which is $\approx 1 / 3 \sqrt{P_{\text {act }} / P_{\text {th }}}$ for $P_{\text {act }} \gg P_{\text {th }}$. If, for example, $P_{\text {act }} \approx 10 P_{\text {th }}$, $\langle b\rangle \approx \Gamma_{\mathrm{act}}$, meaning that, on average, $P_{\mathrm{obs}} \approx P_{\mathrm{act}} / 5$ and $\Gamma_{\mathrm{obs}} \approx 5 \Gamma_{\mathrm{act}}$.

\subsection{The Recovery Bias}

As it travels on the surface of the observational arena, a dust devil's pressure contour $P_{\text {th }}$ carves out a long, narrow area $A\left(P_{\text {act }}, \Gamma_{\text {act }}\right)$. If a barometer lies within that area, the devil will be detected, in principle. $A$ is given by

$$
\begin{aligned}
A & =\pi b_{\max }^{2}+v L b_{\max } \\
& =\left(\Gamma_{\mathrm{act}} / 2\right) \sqrt{\frac{P_{\mathrm{act}}-P_{\mathrm{th}}}{P_{\mathrm{th}}}}\left[\pi\left(\Gamma_{\mathrm{act}} / 2\right) \sqrt{\frac{P_{\mathrm{act}}-P_{\mathrm{th}}}{P_{\mathrm{th}}}}+v L\right],
\end{aligned}
$$

The probability to recover a devil is proportional to this total track area. Thus devils with deeper and wider pressure profiles are more likely to be recovered. Using the lifetime scaling from Lorenz (2014), Figure 5 shows that the second term dominates over the first term for all but the smallest, slowest dust devils, so, for simplicity, we will neglect the first term, giving

$$
A \approx\left(\Gamma_{\mathrm{act}} / 2\right) \sqrt{\frac{P_{\mathrm{act}}-P_{\mathrm{th}}}{P_{\mathrm{th}}}} v L .
$$

The fact that larger, faster dust devils cover more area means that they are more likely to be recovered. We can quantify the recovery probability $f$ by taking the ratio of track areas for a given dust devil to the largest area for a dust devil, $A_{\max }$ :

$$
f=\frac{A\left(\Gamma_{\mathrm{act}}, P_{\mathrm{act}}\right)}{A_{\max }}=A_{\mathrm{max}}^{-1} \Gamma_{\mathrm{act}} \sqrt{\frac{P_{\mathrm{act}}-P_{\mathrm{th}}}{P_{\mathrm{th}}}} v L .
$$




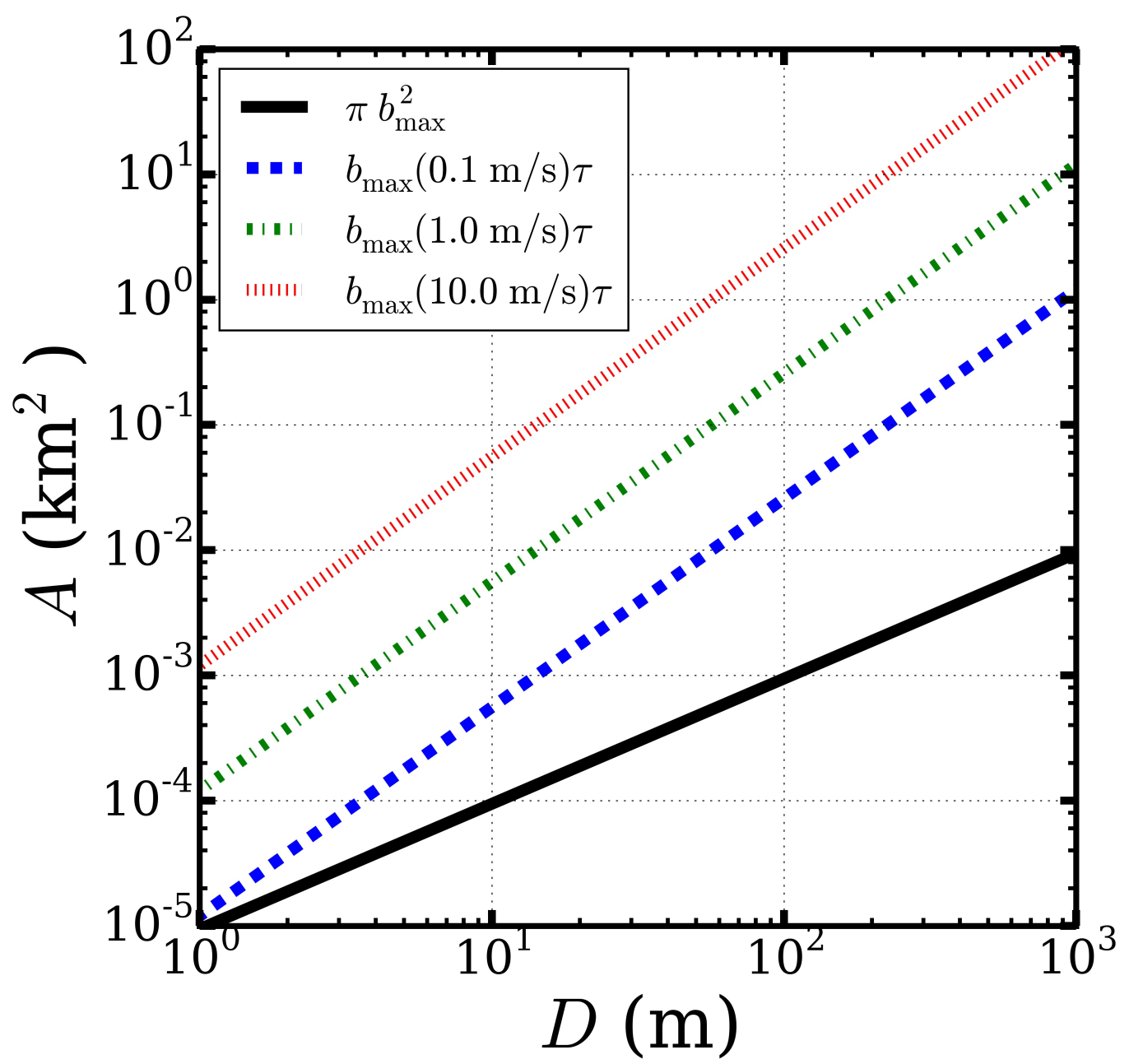

Fig. 5. The two area terms from Equation 12 for dust devils with a range of diameters $D$ traveling with a range of velocities, $0.1,1$, and $10 \mathrm{~m} / \mathrm{s}$.

The devil with the deepest profile need not also have the widest profile or the largest translation velocity. Rennó and Bluestein (2001) argue that the diameter of a vortex is set, in part, by the local vorticity field, while Balme et al. (2012), from their field studies, find no correlation between diameter and translation velocity from their field work. In quantifying the recovery probability $f\left(\Gamma_{\text {act }}, P_{\text {act }}\right)$, it is only important that we apply a uniform normalizing factor to the whole population, so we will take $A_{\max }=\Gamma_{\max } \sqrt{\left(P_{\max }-P_{\mathrm{th}}\right) / P_{\mathrm{th}}} v L_{\max }$, assuming no correlation between translation velocity and diameter or pressure, 
which gives

$$
f=\left[\Gamma_{\max }^{-1} L_{\max }^{-1}\left(P_{\max }-P_{\mathrm{th}}\right)^{-1 / 2}\right] \Gamma_{\mathrm{act}} L\left(P_{\mathrm{act}}-P_{\mathrm{th}}\right)^{1 / 2}
$$

Taking the distribution of observed devils as $\rho\left(\Gamma_{\text {act }}, P_{\text {act }}\right)$, the product $f\left(\Gamma_{\text {act }}, P_{\text {act }}\right) \times$ $\rho\left(\Gamma_{\text {act }}, P_{\text {act }}\right)$ would represent the population of devils that are detected but not how the recovered population would actually look.

\subsection{Converting Between the Observed and Actual Parameter Distributions}

Consider a distribution of observed values $\rho\left(\Gamma_{\text {obs }}, P_{\text {obs }}\right)=d^{2} N / d \Gamma_{\text {obs }} d P_{\text {obs }}$. The small number of devils $d N=f \rho\left(\Gamma_{\text {obs }}, P_{\text {obs }}\right) d \Gamma_{\text {act }} d P_{\text {act }}$ contributing are those that had closest approach distances between $b$ and $b+d b$ of the detector. Thus, we can convert $\rho\left(\Gamma_{\text {act }}, P_{\text {act }}\right)$ to $\rho\left(\Gamma_{\text {obs }}, P_{\text {obs }}\right)$ by integrating $\rho\left(\Gamma_{\text {act }}, P_{\text {act }}\right)$ over $b$ and accounting for the bias and distortion effects:

$$
\rho(\mathrm{obs})=\int_{b^{\prime}=0}^{b(\mathrm{obs})} f \rho\left(\operatorname{act}\left(b^{\prime}\right)\right) \frac{2 b^{\prime} d b^{\prime}}{b_{\max }^{2}},
$$

where $b(\mathrm{obs})=\left(\Gamma_{\mathrm{obs}} / 2\right)\left[\left(P_{\max }-P_{\mathrm{obs}}\right) / P_{\max }\right]^{1 / 2}$, i.e. the maximum radial distance at which the actual distribution could contribute to the observed distribution. Figure 6 shows the result for a uniform distribution of actual values, $\rho($ act $)=\left(P_{\max }-P_{\mathrm{th}}\right)^{-1}\left(\Gamma_{\max }-\Gamma_{\mathrm{th}}\right)^{-1}$ and compares it to the simulated results of an observational survey (blue circles).

Ultimately, we are interested in converting the density of observed parameters to the density of actual parameters. We describe the details of our approach in the Appendix, but the basic idea is that $\rho\left(\Gamma_{\text {obs }}, P_{\text {obs }}\right)$ represents an integral sampling $\rho\left(\Gamma_{\text {act }}, P_{\text {act }}\right)$ along a locus of points $\left(\Gamma_{\text {act }}, P_{\text {act }}\right)$, going from a point representing $b=0$ up to a point representing the maximum radial distance. 

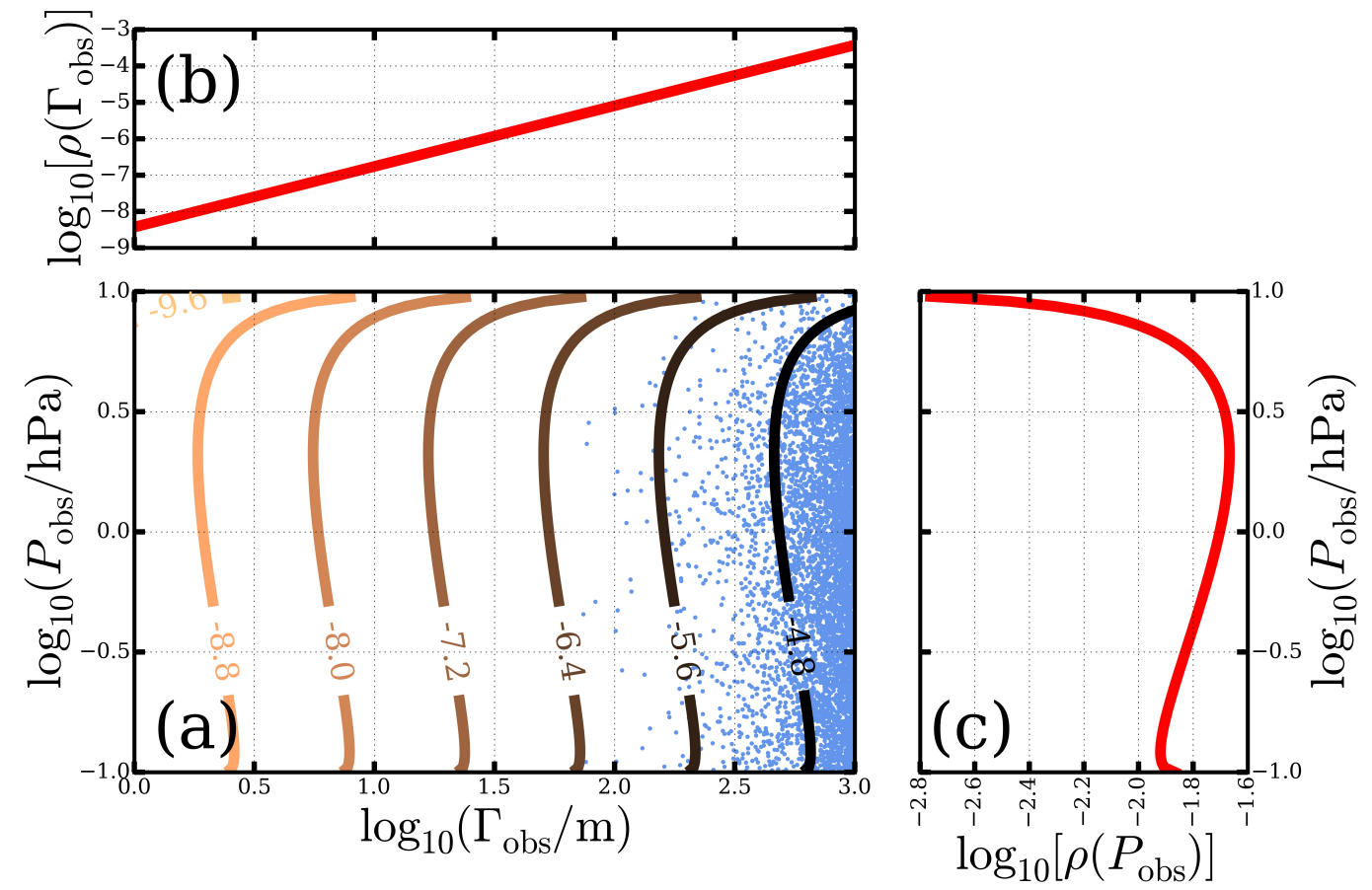

Fig. 6. (a) Contours of number density for observed dust devil parameters, $\log _{10} \rho\left(P_{\text {obs }}, \Gamma_{\text {obs }}\right)$, assuming a uniform distribution of underlying values $\rho\left(P_{\text {act }}, \Gamma_{\text {act }}\right)=\left(P_{\max }-P_{\text {th }}\right)^{-1}\left(\Gamma_{\max }-\Gamma_{\text {th }}\right)^{-1}$. The blue dots show a simulated dust devil survey. (b) Density of $\Gamma_{\text {obs }}$ integrated over $P_{\text {obs }}$. (c) Density of $P_{\text {obs }}$ integrated over $\Gamma_{\text {obs }}$.

Thus, we can differentiate $\rho\left(\Gamma_{\text {obs }}, P_{\text {obs }}\right)$ to determine $\rho\left(\Gamma_{\text {act }}, P_{\text {act }}\right)$ :

$\rho\left(\Gamma_{\text {act }}, P_{\text {act }}\right)=k \Gamma_{\text {act }}^{-5 / 3}\left(P_{\text {act }}-P_{\text {th }}\right)^{-1 / 2}\left(P_{\text {obs }} \frac{\partial \rho\left(\Gamma_{\text {obs }}, P_{\text {obs }}\right)}{\partial P_{\text {obs }}}-\left(\frac{\Gamma_{\text {obs }}}{2}\right) \frac{\partial \rho\left(\Gamma_{\text {obs }}, P_{\text {obs }}\right)}{\partial \Gamma_{\text {obs }}}\right)_{\text {obs } \rightarrow \text { act }}$,

where $k$ is constant and obs $\rightarrow$ act indicates that obs quantities should be replaced with act quantities after the derivatives are taken.

In the next section, we apply Equation 17 to a dataset from a real survey, but as an example, consider the simple differential distribution $\rho\left(\Gamma_{\text {obs }}, P_{\text {obs }}\right)=\alpha P_{\text {obs }}^{-2}$. Applying Equation 17 gives a distribution of actual parameters:

$$
\rho\left(\Gamma_{\text {act }}, P_{\text {act }}\right)=k^{\prime} \Gamma_{\text {act }}^{-5 / 3}\left(P_{\text {act }}-P_{\text {th }}\right)^{-1 / 2} P_{\text {act }}^{-2} .
$$


Note that, for this example, $\partial \rho(\mathrm{obs}) / \partial P_{\text {obs }}<0$. In such a case, the signs on the partial derivatives should be flipped since the limits on the integral for Equation 16 would be flipped.

Equation 18 increases without limit as $P_{\text {act }} \rightarrow P_{\text {th }}$ because such shallow dips are only observed for statistically impossible central encounters $(b=0)$. If we assume $P_{\text {th }} \ll P_{\text {act }}$ for any observed values, then we have:

$$
\rho\left(\Gamma_{\text {act }}, P_{\text {act }}\right) \approx k^{\prime} \Gamma_{\text {act }}^{-5 / 3} P_{\text {act }}^{-5 / 2}
$$

Using a direct numerical simulation of a dust devil barometric survey, Lorenz (2014) found that an observed distribution $\rho\left(P_{\text {obs }}\right) \propto P_{\text {obs }}^{-2}$ required an actual distribution approximately $\rho\left(P_{\text {act }}\right) \propto P_{\text {act }}^{-2.8}$, in line with our results here.

\section{Application to Observational Data}

In this section, we apply our statistical formulation to results from a real survey, Ellehoj et al. (2010), a study using time-series from the Phoenix Lander (Smith et al. 2008). Ellehoj et al. (2010) analyzed 151 sols worth of data, including pressures, temperatures, wind speeds, and images. To identify dust devil passages in the barometric data, they compared the average pressure in a 20-s window to the average pressure in 20-s windows to either side of the former window. Average pressures in the middle window that were different by more than $0.1 \mathrm{~Pa}$ from the average on either side were identified as possible dust devil passages. Then for every pressure event found, the authors analyzed the surrounding pressure and temperature values, and non-significant and false events, e.g., from data transfer gaps, were removed by hand (the precise criteria 
used to exclude an event are not given). In this way, Ellehoj et al. (2010) identified 197 vortices with a pressure drops larger than $0.5 \mathrm{~Pa}=10^{-0.3} \mathrm{~Pa}$, which we will take as $P_{\text {th }}$ for this dataset. Figure 7 shows a scatter plot of their reported detections. The shaded background is calculated using a Gaussian kernel density estimator and a bandwidth of 0.75 , analogous to histogram bin widths (Scott, 1979). To determine the distribution of $\tau_{\text {obs }}$, we integrated the 2-D density over $P_{\text {obs }}$ (i.e., marginalized it), and we show the resulting distribution in the top panel (the right panel shows the analogous result for $\left.P_{\text {obs }}\right)$.

To convert the $\tau_{\text {obs }}$ values to a distribution of $\Gamma_{\text {obs }}$, we used Equation 2 and the wind speeds measured during sols 91 through 150 from the Phoenix mission (Holstein-Rathlou et al., 2010 ${ }^{1}$ (Figure 8 (a)). These wind speeds were measured primarily between 0700 and 1900 local mean solar time, which overlaps with times when dust devils are most common, but they are not all measured contemporaneously to dust devil activity. In other words, the distribution in Figure 8 (a) is broadly representative of daytime wind speeds seen during the Phoenix mission but not necessarily of the wind speeds during dust devil encounters.

Figure 8 (b) shows the resulting $\Gamma_{\text {obs }}$ distribution. Consistent with estimates of the distributions of dust devil widths (e.g. Reiss and Lorenz, 2016), wider devils are less common than narrow devils, and Figure 8 (b) shows a power-law model for the differential distribution with index -1.6 provides a reasonable fit. The distribution also declines for dust devils narrower than $10^{1.25} \mathrm{~m} \approx 18 \mathrm{~m}$,

$\overline{1}$ Available via NASA's Planetary Data System - http://pds-atmospheres. nmsu.edu/pdsd/archive/data/phx-m-tt-5-wind-vel-dir-v10/phxwnd_0001/ DATA/TELLTALE_91_151.TAB 

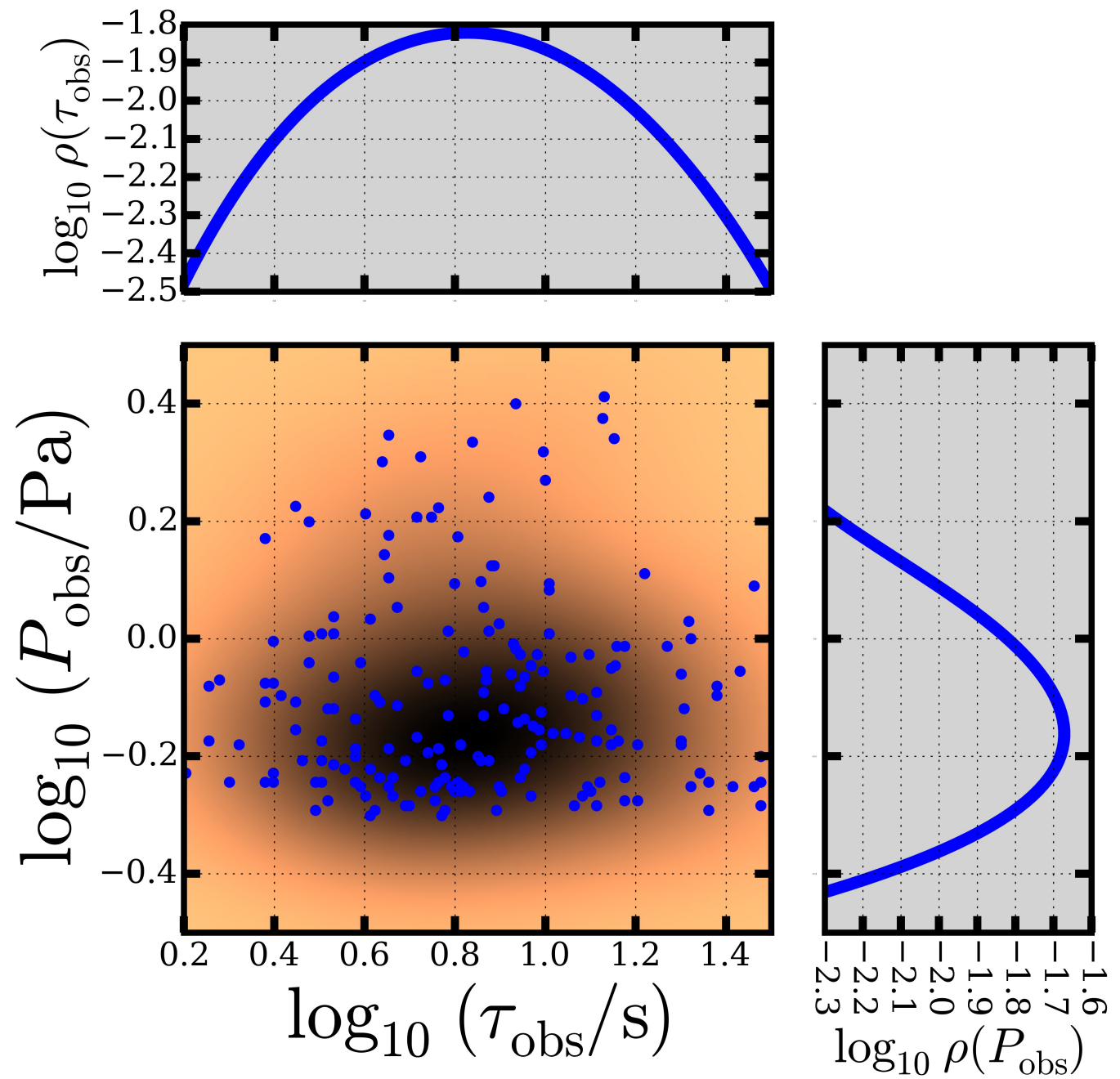

Fig. 7. The bottom left panel shows a scatter plot (blue dots) of Martian dust devil encounters observed by the meteorological instruments on the Pheonix Lander and reported in Table 1 from Ellehoj et al. (2010). The copper-to-black background shows the corresponding density estimated using a Gaussian kernel density estimator, with darker colors indicating higher densities. The blue lines in the top and right panels show the marginalized, normalized distributions of $\tau_{\text {obs }}$ and $P_{\text {obs }}$, respectively.

seemingly at odds with the results of Reiss and Lorenz (2016), which conducted a visual survey of dust devil tracks and reported a population dominated by devils with a diameters $<10 \mathrm{~m}$. However, the apparent decline on the narrow- 

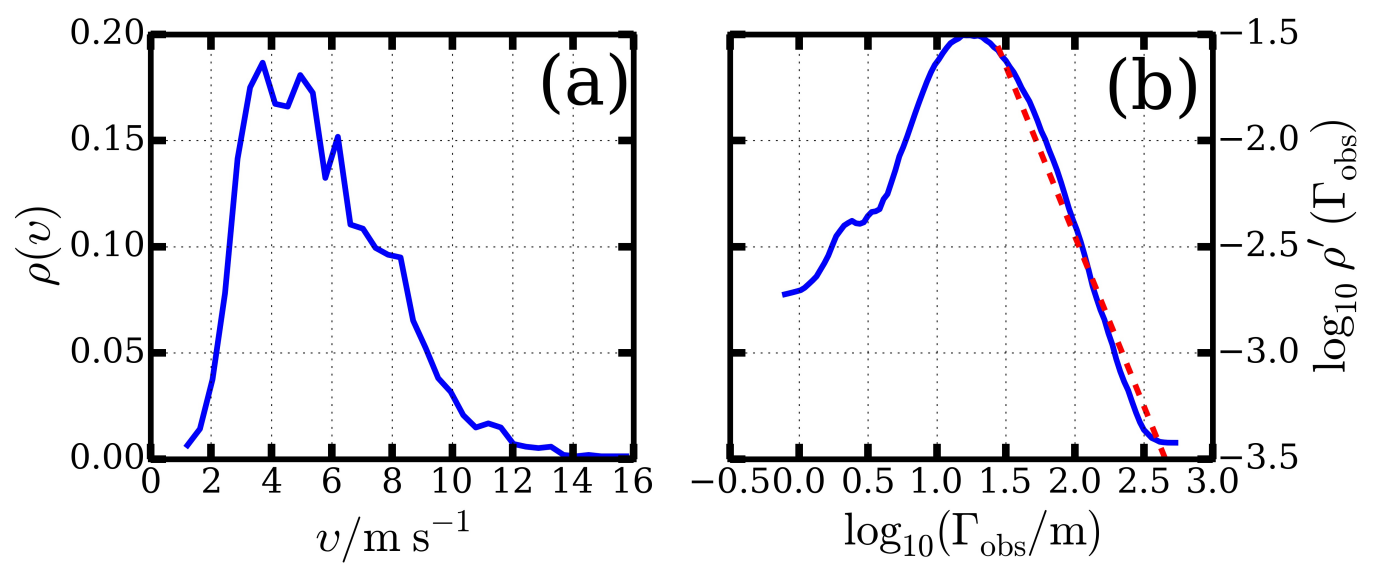

Fig. 8. (a) Distribution of windspeeds measured by the Phoenix mission (Holstein-Rathlou et al., 2010). (b) Inferred normalized distribution of dust devil widths using data from Ellehoj et al. (2010) (solid, blue curve), along with power-law fit having index about -1.6 (dashed, red curve).

end of the distribution is likely the result of selection bias in Ellehoj et al. (2010). Given the typical wind velocity of $5 \mathrm{~m} / \mathrm{s}$ measured at the Phoenix site, a devil with a width $\sim 10 \mathrm{~m}$ would produce a signal with only a $\sim 2$-s duration. The search technique from Ellehoj et al. (2010) requires a devil to induce a deep or lengthy $(\sim 20-\mathrm{s})$ pressure perturbation in order to register a detection, thus filtering out the smallest (and possibly most common) dust devils. Likewise, pressure perturbations spanning much more than $20 \mathrm{~s}$ (presumably, the widest and/or most slowly moving devils) would probably also be filtered out, which may explain why the power-law fit in Figure 8 (b) is shallower than in Reiss and Lorenz (2016).

Finally, we can use Equation 17 to convert the joint distribution of observed parameters $\rho\left(\Gamma_{\text {obs }}, P_{\text {obs }}\right)$ to the distribution of actual parameters $\rho\left(\Gamma_{\text {act }}, P_{\text {act }}\right)$, and the result is shown in Figure 9. The differential distribution for $\Gamma_{\text {act }}$ is slightly shallower than that for $\Gamma_{\text {obs }}$ and is best fit using a power-law index of -1.5 , as compared to -1.6 for $\Gamma_{\text {obs }}$, and the mode of the distribution shifts 
from $18 \mathrm{~m}$ for $\Gamma_{\text {obs }}$ to $13 \mathrm{~m}$ for $\Gamma_{\text {act }}$. The distribution of $P_{\text {act }}$ shifts toward larger pressures compared to the distribution for $P_{\text {obs }}$, with the mode going from 0.7 to $0.9 \mathrm{~Pa}$. As for the distribution of $\Gamma_{\mathrm{obs}}$, selection effects probably suppress the distribution of $P_{\text {obs }}$ for smallest values since devils with smaller pressure dips have a smaller signal-to-noise ratio. For values in the differential distribution of $P_{\text {obs }}$ larger than $0.7 \mathrm{~Pa}$, a power-law model with index about -2 provides a best-fit, while for the $P_{\text {act }}$ distribution, an index of -3.5 provides a best-fit. Keep in mind that this distribution of $P_{\text {act }}$ comes from marginalizing over $\Gamma_{\text {act }}$ the result of applying Equation 17, which is why it is different from the distribution described at the end of the last section.

Since Equation 17 involves a difference between derivatives, it is possible for the inferred distribution of actual values to become negative, even though negative values are not physically meaningful. As we have argued here, the drops in the distributions at small $\Gamma_{\text {obs }}$ and $P_{\text {obs }}$ probably arise from selection effects and are not physical. In order to avoid retrieving negative values for our distributions, we have focused on evaluating Equation 17 in regions where the distribution remains positive, where selection effects are probably less important.

By averaging Equation 11 over the population, we can estimate the populationweighted average miss distance:

$$
\langle\langle b\rangle\rangle=\frac{2}{3} \int b_{\max } / \Gamma_{\text {act }} \rho(\text { act }) d \Gamma_{\text {act }} d P_{\text {act }} \approx 16 \mathrm{~m}
$$

or roughly one full-width/half-max. For this calculation, we only integrated over the population where $\Gamma_{\text {act }} \geq 13 \mathrm{~m}$ and $P_{\text {act }} \geq 0.7 \mathrm{~Pa}$ to mitigate the effects of the detection biases, which likely suppress the observed number of narrow and shallow dust devil signals. 

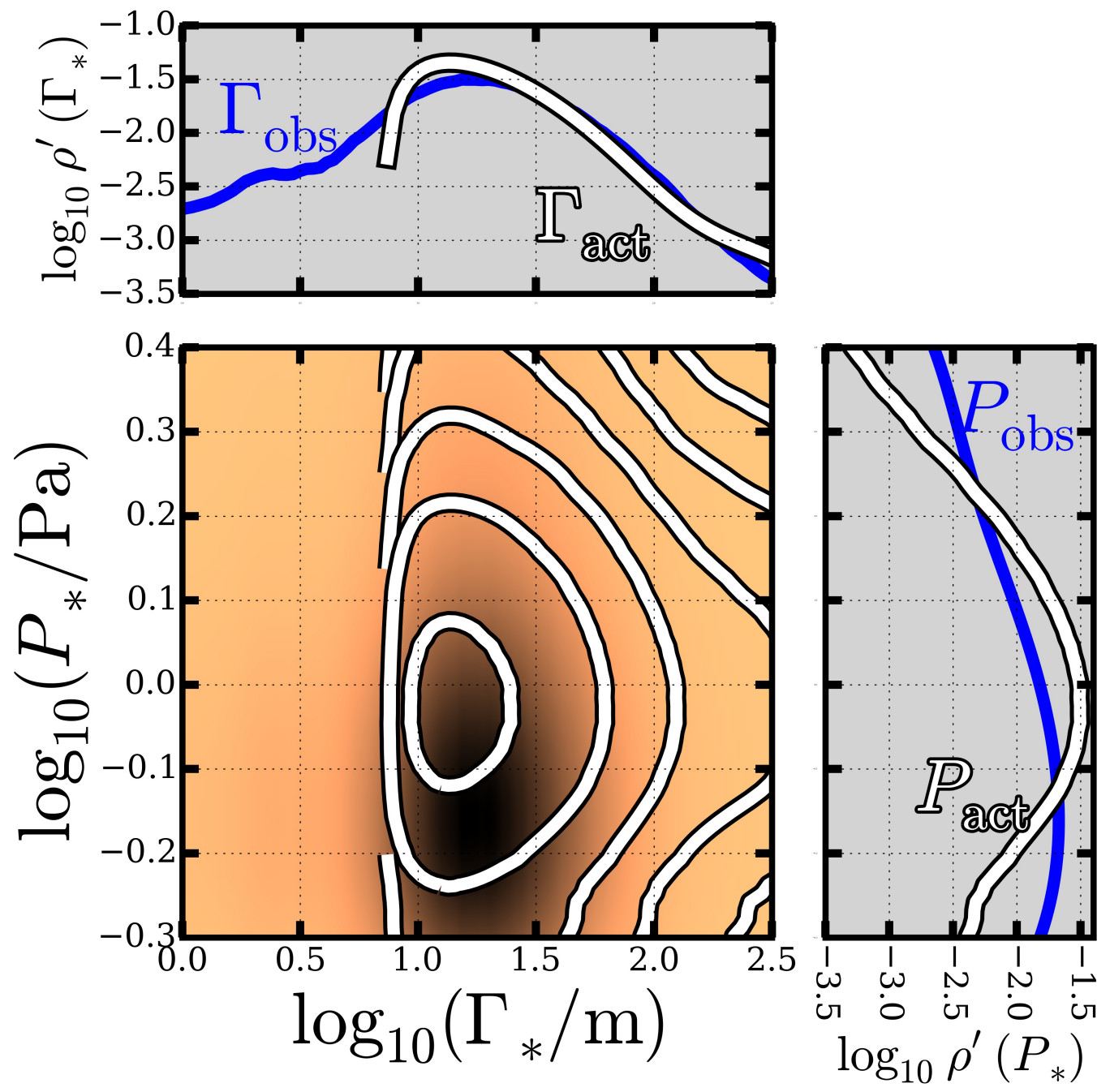

Fig. 9. The conversion between the joint distribution for observed parameters $\rho\left(\Gamma_{\text {obs }}, P_{\text {obs }}\right)$ to that for actual parameters $\rho\left(\Gamma_{\text {act }}, P_{\text {act }}\right)$. The copper-to-black shading in the bottom, left panel shows $\rho\left(\Gamma_{\text {obs }}, P_{\text {obs }}\right)$, with darker colors indicating higher densities, while the white contours show $\rho\left(\Gamma_{\text {act }}, P_{\text {act }}\right)$. The blue lines in the top and right panels show the marginalized, normalized distributions for each of the observed and actual parameters.

\section{Discussion and Conclusions}

Our formulation here provides a starting place for relating the population statistics of dust devils as recovered by single-barometer surveys to their phys- 
ical structures, provided some measure of their translational velocities. Understanding these relationships is critical for understanding the atmospheric influence of devils on both planets since it depends so sensitively on both the devils' statistical and physical properties. As noted in Jackson and Lorenz (2015) and Lorenz (2014), in estimating the total flux of dust injected into the martian atmosphere, it is important to consider the population-weighted flux and not the flux from the average dust devil, which Jackson and Lorenz (2015) showed can amount to several orders of magnitude increase in the dust flux. The bias/distortion corrections to the populations discussed here increases the flux by at least $20 \%$ over that. Accounting for biases in the time-series analysis could increase the flux even more since, as discussed here, the scheme used in Holstein-Rathlou et al. (2010) likely suppresses the recovery of the larger/slowmoving vortices, which may lift the most dust, and smaller/quickly moving dust devils, which are the most numerous.

As discussed in Section 2.3, the miss distance effect biases the recovered population toward the physically widest devils. Because the dynamical processes that form and maintain devils are not well-understood, the relationship between the width of a devil and its other physical properties are not clear. Fenton and Lorenz (2015) argue that dust devil height is related to the boundary layer depth, while the physical model outlined in Rennó et al. (1998) indicates the profile depth should also scale with boundary layer depth. In any case, the bias definitely plays a role in estimates of the areal density for dust devil occurrence. For example, by assuming a devil profile width of $100 \mathrm{~m}$, Ellehoj et al. (2010) combine the number of devils recovered from pressure time-series and wind speed data to estimate a local occurrence rate of 1 event per sol per $10 \mathrm{~km}^{2}$. Our analysis here (Figure 8) shows that such wide devils were 
probably not typical among those detected. Instead, we can adapt their formulation for our estimated typical, actual diameter of 13 m (Figure 9), which gives an occurrence rate ten times higher, 1 event per sol per $\mathrm{km}^{2}$. Investigating the abundance of dust devil tracks in the southern hemisphere in Argyre Planitia and Hellas Basin, Balme et al. (2003b) estimated rates as high as about 0.2 events per sol per $\mathrm{km}^{2}$, suggesting that something like only one in five low-pressure cells lift sufficient dust to leave a visible track. This estimate includes detection biases and a population average should be used instead of typical values, but these results are roughly consistent with terrestrial field studies that estimate $40 \%$ of dust devils lift visible amounts of dust Lorenz and Jackson, 2015).

The model for the miss distance effect developed here serves to highlight the many important uncertainties and degeneracies involved in single-barometer dust devil surveys, in particular, the difficulty of disentangling the geometry of an encounter between a devil and a detector from the devil's structure. However, the encounter geometry can be determined if additional measurements are made. For instance, if the tangential velocity profile for a dust devil can be measured simultaneously with the pressure profile, the miss distance can be determined directly by assuming cyclostrophic balance, i.e. $V_{\mathrm{T}}^{2}=\Delta P_{\text {act }} / \rho$, a pressure profile given by Equation 1, and one of the physically motivated vortex profiles given in Vatistas et al. (1991) for the tangential windspeed:

$$
V(r)=\frac{2 r V_{\mathrm{T}}}{1+\left(2 r / \Gamma_{\mathrm{act}}\right)^{2}},
$$

where $V_{\mathrm{T}}$ is the windspeed at the eyewall of the vortex and $\rho$ is the atmospheric density. With these assumptions, the pressure $P$ and velocity $V$ measured at any point in the profile obey 


$$
\begin{aligned}
P_{\mathrm{act}} & =\left(1-\frac{\rho V^{2}}{4 P}\right)^{-1} P, \text { and } \\
V_{\mathrm{T}} & =\left(1-\frac{\rho V^{2}}{4 P}\right)^{-1 / 2}\left(\frac{P}{\rho}\right)^{1 / 2} .
\end{aligned}
$$

Unfortunately, many in-situ surveys produce only temperature time-series along with the pressure time-series, but a clear prediction of the temperature profile might allow a determination of the miss distance directly from the pressure and temperature time-series.

An improved understanding of the biases involved in a detection scheme is critical for relating the observed to the underlying population, and a simple way to assess a scheme's detection efficiency is to inject synthetic devil signals (with known parameters) into the real data streams. Then the detection scheme can be applied to recover the synthetic devils and the efficiency of detection assessed across a swath of devil parameters. Such an approach is common in exoplanet transit searches (e.g. Sanchis-Ojeda et al., 2014), where dips in photometric time series from planetary shadows closely resemble dust devil pressure signals. By injecting synthetic devils into real data, the often complex noise structure in the data is retained and simplifying assumptions (such as stationary white noise) are not required.

Among important limitations of our model, the translation velocity $v$ for devils remains a critical uncertainty for relating physical and statistical properties. This limitation points to the need for wind velocity measurements made simultaneously with pressure measurements in order to accurately estimate dust devil widths. In particular, correlations between $v$ and dust devil properties will skew the recovered parameters in ways not captured here. For example, the devils with the deepest pressure profiles seem to occur preferentially 
around mid-day local time both on Mars Ellehoj et al. (2010) and the Earth Jackson and Lorenz (2015). If winds at that time of day are preferentially fast or slow, then the profile widths recovered for the deepest devils will be skewed toward smaller or larger values. In addition, some field observations suggest devils with larger diameters may be advected more slowly than their smaller counterparts Greeley et al. (2010), which would tend to make their profiles look wider.

Clear predictions of the distributions of physical parameters for dust devils from high resolution meteorological models would be especially helpful for constraining and directing this work, and some progress in this area has been made. For example, Kanak (2005) applied a large-eddy simulation of a planetary convective boundary layer to study vortical structures and the influence of ambient conditions on their formation. For the handful of vortices formed in the simulations, there was good qualitative agreement with observation. Gheynani and Taylor (2010) also studied vortex formation on Earth and Mars and noted the role of the boundary layer's depth on vortex scale. Given the stochastic nature of boundary layer dynamics, detailed statistical predictions from such models are needed for comparison to observation. Recently, Nishizawa et al. (2016) have systematically explored size-frequency distributions in a large eddy simulation run at different resolutions.

Likely the best way to study dust devil formation and dynamics in the field is not statistically, but directly via deployment of sensor networks that produce a variety of data streams with high spatial and time resolution. Field work with in-situ sensors has a long history but usually involving single-site deployments (e.g. Sinclair, 1973). In the decades since that study, technological developments in miniaturization and data storage now provide a wealth of 
robust and inexpensive instrumentation, ideally suited for the long-term field deployment required to study dust devils, without the need for direct human involvement. Recently, Lorenz et al. (2015) deployed an array of ten miniature pressure- and sunlight-logging stations at La Jornada Experimental Range in New Mexico, providing a census of vortex and dust-devil activity at this site. The simultaneous measurements resolved horizontal pressure structures for several dust devils, giving entirely independent estimates of vortex size and intensity.

The rich and growing databases of high-time-resolution meteorological data, both for the Earth and Mars, combined with the wide availability and affordability of robust instrumentation, point to bright future for dust devil studies. The data streaming in from the Mars Science Laboratory Rover Environmental Monitoring Station (REMS) (Gómez-Elvira et al., 2012) may provide new insight into Martian dust devils, and recent studies using imaging data from the Mars Science Laboratory (e.g. Lemmon et al., 2017) have spotted many dust devils in Gale Crater. The formulation presented here provides a simple but robust scheme for relating the dust devils' statistical and physical properties, and it represents an important next step in improving our knowledge of these dynamic and ethereal phenomena.

\section{Acknowledgments}

The authors acknowledge helpful conversations with Leming $\mathrm{Qu}$ and Paul Simmonds. This research was supported, in part, by the Idaho Space Grant Consortium. 


\section{References}

M. Balme, S. Metzger, M. Towner, T. Ringrose, R. Greeley, and J. Iversen. Friction wind speeds in dust devils: A field study. Geophysical Research Letters, 30(16), 2003a. doi: 10.1029/2003gl017493. URL http://dx.doi. org/10.1029/2003gl017493.

M. R. Balme, P. L. Whelley, and R. Greeley. Mars: Dust devil track survey in Argyre Planitia and Hellas Basin. Journal of Geophysical Research (Planets), 108:5086, August 2003b. doi: 10.1029/2003JE002096.

Matt Balme and Ronald Greeley. Dust devils on Earth and Mars. Rev. Geophys., 44(3), 2006. doi: 10.1029/2005rg000188. URL http://dx.doi. org/10.1029/2005rg000188.

M.R. Balme, A. Pathare, S.M. Metzger, M.C. Towner, S.R. Lewis, A. Spiga, L.K. Fenton, N.O. Renno, H.M. Elliott, F.A. Saca, T.I. Michaels, P. Russell, and J. Verdasca. Field measurements of horizontal forward motion velocities of terrestrial dust devils: Towards a proxy for ambient winds on Mars and Earth. Icarus, 221(2):632-645, nov 2012. doi: 10.1016/j.icarus.2012.08.021. URL http://dx.doi.org/10.1016/j.icarus.2012.08.021.

Shabari Basu. Simulation of the Martian dust cycle with the GFDL Mars GCM. J. Geophys. Res., 109(E11), 2004. doi: 10.1029/2004je002243. URL http://dx.doi.org/10.1029/2004je002243.

M. D. Ellehoj, H. P. Gunnlaugsson, P. A. Taylor, H. Kahanp, K. M. Bean, B. A. Cantor, B. T. Gheynani, L. Drube, D. Fisher, A.-M. Harri, C. HolsteinRathlou, M. T. Lemmon, M. B. Madsen, M. C. Malin, J. Polkko, P. H. Smith, L. K. Tamppari, W. Weng, and J. Whiteway. Convective vortices and dust devils at the Phoenix Mars mission landing site. J. Geophys. Res., 115, 2010. doi: 10.1029/2009je003413. URL http://dx.doi.org/10.1029/ 
Lori K. Fenton and Ralph Lorenz. Dust devil height and spacing with relation to the martian planetary boundary layer thickness. Icarus, 260:246-262, nov 2015. doi: 10.1016/j.icarus.2015.07.028. URL http://dx.doi .org/10. 1016/j.icarus.2015.07.028.

B. T. Gheynani and P. A. Taylor. Large-Eddy Simulations of Vertical Vortex Formation in the Terrestrial and Martian Convective Boundary Layers. Boundary-Layer Meteorology, 137:223-235, nov 2010. doi: 10.1007/ s10546-010-9530-z.

Dale A. Gillette and Peter C. Sinclair. Estimation of suspension of alkaline material by dust devils in the United States. Atmospheric Environment. Part A. General Topics, 24(5):1135-1142, jan 1990. doi: 10.1016/0960-1686(90) 90078-2. URL http://dx.doi.org/10.1016/0960-1686(90)90078-2.

J. Gómez-Elvira, C. Armiens, L. Castañer, M. Domínguez, M. Genzer, F. Gómez, R. Haberle, A.-M. Harri, V. Jiménez, H. Kahanp, L. Kowalski, A. Lepinette, J. Martín, J. Martínez-Frías, I. McEwan, L. Mora, J. Moreno, S. Navarro, M. A. de Pablo, V. Peinado, A. Peña, J. Polkko, M. Ramos, N. O. Renno, J. Ricart, M. Richardson, J. Rodríguez-Manfredi, J. Romeral, E. Sebastián, J. Serrano, M. de la Torre Juárez, J. Torres, F. Torrero, R. Urquí, L. Vázquez, T. Velasco, J. Verdasca, M.-P. Zorzano, and J. Martín-Torres. REMS: The Environmental Sensor Suite for the Mars Science Laboratory Rover. Space Science Reviews, 170(1-4):583-640, aug 2012. doi: 10.1007/s11214-012-9921-1. URL http://dx.doi.org/10. 1007/s11214-012-9921-1.

Ronald Greeley, Devin A. Waller, Nathalie A. Cabrol, Geoffrey A. Landis, Mark T. Lemmon, Lynn D. V. Neakrase, Mary Pendleton Hoffer, Shane D. Thompson, and Patrick L. Whelley. Gusev Crater Mars: Ob- 
servations of three dust devil seasons. J. Geophys. Res., 115, 2010. doi: 10.1029/2010je003608. URL http://dx.doi.org/10.1029/2010JE003608. C. Holstein-Rathlou, H. P. Gunnlaugsson, J. P. Merrison, K. M. Bean, B. A. Cantor, J. A. Davis, R. Davy, N. B. Drake, M. D. Ellehoj, W. Goetz, S. F. Hviid, C. F. Lange, S. E. Larsen, M. T. Lemmon, M. B. Madsen, M. Malin, J. E. Moores, P. Nørnberg, P. Smith, L. K. Tamppari, and P. A. Taylor. Winds at the Phoenix landing site. Journal of Geophysical Research (Planets), 115:E00E18, May 2010. doi: 10.1029/2009JE003411.

Brian Jackson and Ralph Lorenz. A multiyear dust devil vortex survey using an automated search of pressure time series. J. Geophys. Res. Planets, 120 (3):401-412, mar 2015. doi: 10.1002/2014je004712. URL http://dx.doi. org/10.1002/2014JE004712.

K. M. Kanak. Numerical simulation of dust devil-scale vortices. Quarterly Journal of the Royal Meteorological Society, 131:1271-1292, apr 2005. doi: 10.1256/qj.03.172.

M. V. Kurgansky, R. D. Lorenz, N. O. Renno, T. Takemi, Z. Gu, and W. Wei. Dust Devil Steady-State Structure from a Fluid Dynamics Perspective. Space Sci. Rev., 203:209-244, November 2016. doi: 10.1007/ s11214-016-0281-0.

M. T. Lemmon, C. E. Newman, N. Renno, E. Mason, M. Battalio, M. I. Richardson, and H. Kahanpää. Dust Devil Activity at the Curiosity Mars Rover Field Site. In Lunar and Planetary Science Conference, volume 48 of Lunar and Planetary Science Conference, page 2952, March 2017.

R. Lorenz and M. Myers. Dust Devil Hazard to Aviation: A Review of United States Air Accident Reports. J. Meteor., 30(298):178-184, feb 2005.

R. Lorenz and D. Reiss. Solar Panel Clearing Events, Dust Devil Tracks, and in-situ Vortex Detections on Mars, Icarus, 248,162164. Icarus, 248(0): 
162-164, feb 2014.

R. D. Lorenz. Heuristic estimation of dust devil vortex parameters and trajectories from single-station meteorological observations: Application to InSight at Mars. Icarus, 271:326-337, June 2016. doi: 10.1016/j.icarus.2016. 02.001 .

R. D. Lorenz and B. K. Jackson. Dust Devil Populations and Statistics. Space Sci. Rev., 203:277-297, November 2016. doi: 10.1007/ s11214-016-0277-9.

R. D. Lorenz, L. D. Neakrase, and J. D. Anderson. In-situ measurement of dust devil activity at La Jornada Experimental Range, New Mexico, USA. Aeolian Research, 19:183-194, December 2015. doi: 10.1016/j.aeolia.2015. 01.012 .

Ralph Lorenz. On the statistical distribution of dust devil diameters. Icarus, 215(1):381-390, sep 2011. doi: 10.1016/j.icarus.2011.06.005. URL http: //dx.doi.org/10.1016/j.icarus.2011.06.005.

Ralph Lorenz. The longevity and aspect ratio of dust devils: Effects on detection efficiencies and comparison of landed and orbital imaging at Mars. Icarus, 226(1):964-970, sep 2013. doi: 10.1016/j.icarus.2013.06.031. URL http://dx.doi.org/10.1016/j.icarus.2013.06.031.

Ralph D. Lorenz. Power law of dust devil diameters on Mars and Earth. Icarus, 203(2):683-684, oct 2009. doi: 10.1016/j.icarus.2009.06.029. URL http://dx.doi.org/10.1016/j.icarus.2009.06.029.

Ralph D. Lorenz. Power law distribution of pressure drops in dust devils: Observation techniques and Earth-Mars comparison. Planetary and Space Science, 60(1):370-375, jan 2012. doi: 10.1016/j.pss.2011.11.003. URL http://dx.doi.org/10.1016/j.pss.2011.11.003.

Ralph D. Lorenz. Vortex Encounter Rates with Fixed Barometer Stations: 
Comparison with Visual Dust Devil Counts and Large-Eddy Simulations. J. Atmos. Sci., 71(12):4461-4472, dec 2014. doi: 10.1175/jas-d-14-0138.1. URL http://dx .doi .org/10.1175/JAS-D-14-0138.1.

Ralph D. Lorenz and Brian K. Jackson. Dust devils and dustless vortices on a desert playa observed with surface pressure and solar flux logging. GeoResJ, 5:1-11, mar 2015. doi: 10.1016/j.grj.2014.11.002. URL http: //dx.doi.org/10.1016/j.grj.2014.11.002.

J. Murphy, K. Steakley, M. Balme, G. Deprez, F. Esposito, H. Kahanpää, M. Lemmon, R. Lorenz, N. Murdoch, L. Neakrase, M. Patel, and P. Whelley. Field Measurements of Terrestrial and Martian Dust Devils. Space Sci. Rev., 203:39-87, November 2016. doi: 10.1007/s11214-016-0283-y.

Lynn D. V. Neakrase, Ronald Greeley, James D. Iversen, M. R. Balme, and Eric E. Eddlemon. Dust flux within dust devils: Preliminary laboratory simulations. Geophys. Res. Lett., 33(19), 2006. doi: 10.1029/2006gl026810. URL http: //dx.doi .org/10.1029/2006GL026810.

S. Nishizawa, M. Odaka, Y. O. Takahashi, K.-i. Sugiyama, K. Nakajima, M. Ishiwatari, S.-i. Takehiro, H. Yashiro, Y. Sato, H. Tomita, and Y.Y. Hayashi. Martian dust devil statistics from high-resolution largeeddy simulations. Geophys. Res. Lett., 43:4180-4188, May 2016. doi: 10.1002/2016GL068896.

A.V. Pathare, M.R. Balme, S.M. Metzger, A. Spiga, M.C. Towner, N.O. Renno, and F. Saca. Assessing the power law hypothesis for the size-frequency distribution of terrestrial and martian dust devils. Icarus, 209(2):851-853, oct 2010. doi: 10.1016/j.icarus.2010.06.027. URL http: //dx.doi.org/10.1016/j.icarus.2010.06.027.

D. Reiss and R. D. Lorenz. Dust devil track survey at Elysium Planitia, Mars: Implications for the InSight landing sites. Icarus, 266:315-330, March 2016. 
doi: 10.1016/j.icarus.2015.11.012.

Nilton O. Rennó and Howard B. Bluestein. A Simple Theory for Waterspouts. J. Atmos. Sci., 58(8):927-932, apr 2001. doi: 10.1175/ 1520-0469(2001)058<0927:astfw $>2.0 . c o ; 2 . \quad$ URL http://dx.doi.org/10. 1175/1520-0469(2001)058<0927: ASTFW>2.0.C0;2.

Nilton O. Rennó, Matthew L. Burkett, and Matthew P. Larkin. A Simple Thermodynamical Theory for Dust Devils. J. Atmos. Sci., 55(21):32443252, nov 1998. doi: 10.1175/1520-0469(1998)055〈3244:asttfd $>2.0 . c 0 ; 2$. URL http://dx.doi.org/10.1175/1520-0469(1998)055<3244:ASTTFD> 2.0.CO;2.

Roberto Sanchis-Ojeda, Saul Rappaport, Joshua N. Winn, Michael C. Kotson, Alan Levine, and Ileyk El Mellah. A STUDY OF THE SHORTESTPERIOD PLANETS FOUND WITH KEPLER. ApJ, 787(1):47, may 2014. doi: 10.1088/0004-637x/787/1/47. URL http://dx.doi.org/10.1088/ $0004-637 X / 787 / 1 / 47$.

David W. Scott. On optimal and data-based histograms. Biometrika, 66(3): 605-610, 1979. doi: 10.1093/biomet/66.3.605. URL http://dx.doi.org/ 10.1093/biomet/66.3.605.

Peter C. Sinclair. The Lower Structure of Dust Devils. J. Atmos. Sci., 30 (8):1599-1619, nov 1973. doi: 10.1175/1520-0469(1973)030<1599:tlsodd $\rangle 2$. 0.co;2. URL http://dx.doi.org/10.1175/1520-0469(1973)030<1599: tlsodd $>2.0 . \operatorname{co} ; 2$.

P. H. Smith, L. Tamppari, R. E. Arvidson, D. Bass, D. Blaney, W. Boynton, A. Carswell, D. Catling, B. Clark, T. Duck, E. DeJong, D. Fisher, W. Goetz, P. Gunnlaugsson, M. Hecht, V. Hipkin, J. Hoffman, S. Hviid, H. Keller, S. Kounaves, C. F. Lange, M. Lemmon, M. Madsen, M. Malin, W. Markiewicz, J. Marshall, C. McKay, M. Mellon, D. Michelangeli, 
D. Ming, R. Morris, N. Renno, W. T. Pike, U. Staufer, C. Stoker, P. Taylor, J. Whiteway, S. Young, and A. Zent. Introduction to special section on the Phoenix Mission: Landing Site Characterization Experiments Mission Overviews, and Expected Science. J. Geophys. Res., 113, 2008. doi: 10.1029/2008je003083. URL http://dx.doi.org/10.1029/2008JE003083.

K. Steakley and J. R. Murphy. Investigating Dust-less Devils at Gale Crater. In AAS/Division for Planetary Sciences Meeting Abstracts, volume 46 of AAS/Division for Planetary Sciences Meeting Abstracts, page 300.06, nov 2014 .

P. Thomas and P. J. Gierasch. Dust Devils on Mars. Science, 230(4722):175177, oct 1985. doi: 10.1126/science.230.4722.175. URL http://dx.doi. org/10.1126/science.230.4722.175.

G. H. Vatistas, V. Kozel, and W. C. Mih. A simpler model for concentrated vortices. Experiments in Fluids, 11(1):73-76, apr 1991. doi: 10.1007/bf00198434. URL http://dx.doi.org/10.1007/BF00198434.

M.-P. Zorzano, F. J. Martín-Torres, H. Kahanpää, J. Moore, S. Navarro, A. Lepinette, E. Sebastian, J. Gómez-Elvira, REMS Team, and MSL Science Team. Radiation obscuration by dust devils at Gale as observed by the REMS UV Sensor. In EGU General Assembly Conference Abstracts, volume 15 of EGU General Assembly Conference Abstracts, page 11155, apr 2013. 


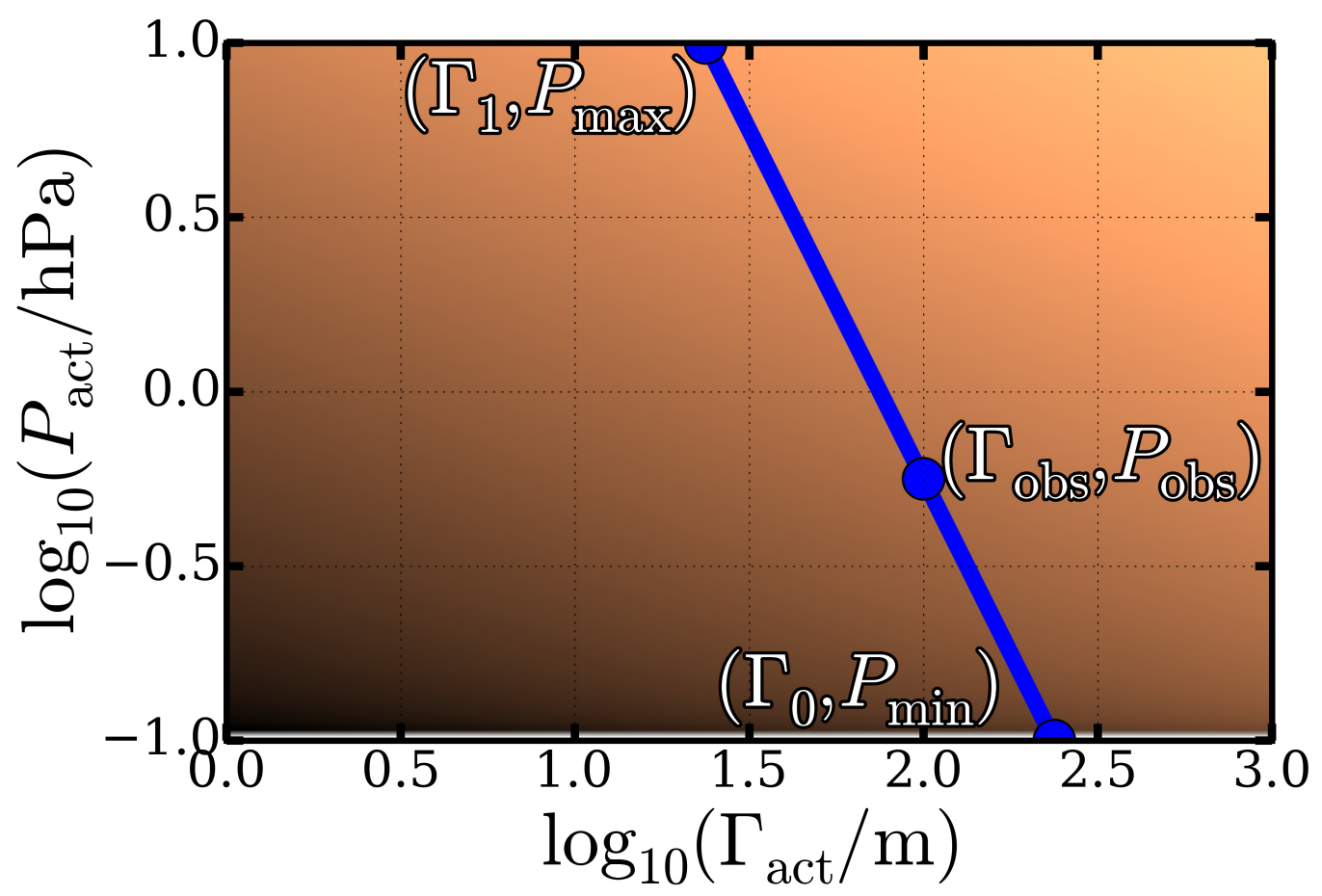

Fig. 10. Example of an integration track for Equation 16. The shading illustrates an example $\rho\left(\Gamma_{\text {act }}, P_{\text {act }}\right)$ distribution, discussed below - brighter shades represent smaller densities. The blue track represents the locus of points passing through given $\Gamma_{\text {obs }}$ and $P_{\text {obs }}$ values. For example, $\rho\left(\Gamma_{\text {obs }}, P_{\text {obs }}\right)$ involves integrating from $b=0$, where $\left(\Gamma_{\text {act }}, P_{\text {act }}\right)=\left(\Gamma_{\text {obs }}, P_{\text {obs }}\right)$ up to a maximum $b$ value, at $\left(\Gamma_{\text {act }}, P_{\text {act }}\right)=\left(\Gamma_{1}, P_{\max }\right)$.

\section{Appendix}

First, we provide the distribution of $\Gamma$ values described in Section 2.1: 


$$
\rho(\Gamma)= \begin{cases}k \alpha^{-1}\left[\tau_{\min }^{-\alpha}-\tau_{\max }^{-\alpha}\right], & \text { if } \Gamma<v_{\max } \tau_{\min } \& \Gamma<v_{\min } \tau_{\max } \\ k \alpha^{-1}\left[\tau_{\min }^{-\alpha}-\left(\Gamma / v_{\min }\right)^{-\alpha}\right], & \text { if } \Gamma<v_{\max } \tau_{\min } \& \Gamma>v_{\min } \tau_{\max } \\ k\left[\left(\alpha^{-1}-\left(\frac{v_{\max }-v_{\min }}{v_{\max }}\right)\right)\left(\Gamma / v_{\max }\right)^{-\alpha}-\tau_{\max }^{-\alpha}\right], & \text { if } \Gamma>v_{\max } \tau_{\min } \& \Gamma<v_{\min } \tau_{\max } \\ k\left[\alpha^{-1}\left(\Gamma / v_{\min }\right)^{-\alpha}-\left(\alpha^{-1}-\frac{v_{\max }-v_{\min }}{v_{\max }}\right)\left(\Gamma / v_{\max }\right)^{-\alpha}\right], & \text { if } \Gamma>v_{\max } \tau_{\min } \& \Gamma>v_{\min } \tau_{\max } .\end{cases}
$$

Next, we discuss the details of Equation 17. Figure 10 illustrates the integration track involved in Equation 16, and we define the end points of the integration track as $\Gamma_{0} \equiv \Gamma_{\text {obs }}\left(P_{\text {obs }} / P_{\text {th }}\right)^{1 / 2}$ and $\Gamma_{1} \equiv \Gamma_{\text {obs }}\left(P_{\text {obs }} / P_{\max }\right)^{1 / 2}$. The equation involves an integral over $b$, which, given $\Gamma_{\text {obs }}$ and $P_{\text {obs }}$, represents a fixed curve in $\Gamma_{\text {act }}-P_{\text {act }}$. In other words, $\Gamma_{\text {obs }}$ and $P_{\text {obs }}$ define a locus of points for $\Gamma_{\text {act }}$ and $P_{\text {act }}$, and the integral over $b$ involves traveling along the locus from the point $\left(\Gamma_{\text {act }}, P_{\text {act }}\right)=\left(\Gamma_{\text {obs }}, P_{\text {obs }}\right)$ up to $\left(\Gamma_{1}, P_{\text {max }}\right)$. In fact, any points $\left(\Gamma_{\text {obs }}^{\prime}, P_{\text {obs }}^{\prime}\right)$ satisfying Equation 8, $P_{\text {obs }}^{\prime} \Gamma_{\text {obs }}^{\prime 2}=P_{\text {obs }} \Gamma_{\text {obs }}^{2}$, lie on this locus. Consequently, the only difference between $\rho\left(\Gamma_{\text {obs }}^{\prime}, P_{\text {obs }}^{\prime}\right)$ and $\rho\left(\Gamma_{\text {obs }}, P_{\text {obs }}\right)$ is where on the track the integral starts - the integrals for both end at the same point:

$$
\begin{aligned}
\rho\left(\Gamma_{\mathrm{obs}}, P_{\mathrm{obs}}\right)-\rho\left(\Gamma_{0}, P_{\mathrm{th}}\right) & =\int_{\left(\Gamma_{\mathrm{obs}}, P_{\mathrm{obs}}\right)}^{\left(\Gamma_{1}, P_{\max }\right)} \cdots d b^{\prime}-\int_{\left(\Gamma_{0}, P_{\mathrm{th}}\right)}^{\left(\Gamma_{1}, P_{\max }\right)} \cdots d b^{\prime} \\
& =\int_{\left(\Gamma_{0}, P_{\mathrm{th}}\right)}^{\left(\Gamma_{\mathrm{obs}}, P_{\mathrm{obs}}\right)} \cdots d b^{\prime}=\int_{b^{\prime}=0}^{b} \cdots d b^{\prime},
\end{aligned}
$$

where we have suppressed the integrands for clarity. We can then differentiate both sides with respect to $b=\left(\Gamma_{\mathrm{obs}} / 2\right)\left[\left(P_{\mathrm{obs}}-P_{\mathrm{th}}\right) / P_{\mathrm{th}}\right]^{1 / 2}$, but, for the lefthand side, we will convert the $b$-derivative: 


$$
\begin{aligned}
\frac{d}{d b} & =\left(\frac{d P_{\mathrm{obs}}}{d b}\right) \frac{\partial}{\partial P_{\mathrm{obs}}}-\left(\frac{d \Gamma_{\mathrm{obs}}}{d b}\right) \frac{\partial}{\partial \Gamma_{\mathrm{obs}}} \\
& =\left(\frac{2}{\Gamma_{\mathrm{obs}}}\right)\left(\frac{P_{\mathrm{obs}}-P_{\mathrm{th}}}{P_{\mathrm{th}}}\right)^{-1 / 2}\left(P_{\mathrm{obs}} \frac{\partial}{\partial P_{\mathrm{obs}}}-\left(\frac{\Gamma_{\mathrm{obs}}}{2}\right) \frac{\partial}{\partial \Gamma_{\mathrm{obs}}}\right) .
\end{aligned}
$$

Thus,

$$
\frac{d}{d b}\left(\rho\left(\Gamma_{\mathrm{obs}}, P_{\mathrm{obs}}\right)-\rho\left(\Gamma_{0}, P_{\mathrm{th}}\right)\right)=\frac{d}{d b}\left(\int_{b^{\prime}=0}^{b} f \rho(\text { act }) \frac{2 b^{\prime} d b^{\prime}}{b_{\max }^{2}}\right)
$$

giving

$$
\rho\left(\Gamma_{\text {act }}, P_{\text {act }}\right)=k \Gamma_{\text {act }}^{-5 / 3}\left(P_{\text {act }}-P_{\text {th }}\right)^{-1 / 2}\left(P_{\text {obs }} \frac{\partial \rho\left(\Gamma_{\text {obs }}, P_{\text {obs }}\right)}{\partial P_{\text {obs }}}-\left(\frac{\Gamma_{\text {obs }}}{2}\right) \frac{\partial \rho\left(\Gamma_{\text {obs }}, P_{\text {obs }}\right)}{\partial \Gamma_{\text {obs }}}\right)_{\text {obs } \rightarrow \text { act }} .
$$

